\title{
Adiabatic Following and Slow Optical Pulse Propagation in Rubidium Vapor
}

\author{
D. Grischkowsky \\ IBM Thomas J. Watson Research Center, Yorktown Heights, New York 10598 \\ (Received 14 November 1972; revised manuscript received 5 February 1973)
}

\begin{abstract}
Short pulses of narrow-line low-intensity dye-laser light nearly resonant with the Zeeman-split ${ }^{2} P_{1 / 2}$ resonance line $(7948 \AA)$ of rubidium were observed to propagate through dilute rubidium vapor as slowly as $(1 / 14) c$. These slow pulse velocities showed that most of the energy in the propagating wave was contained by the vapor as coherent atomic excitation. The observed pulse velocities $v_{p}$ are in good agreement with the equation $v_{p}=d \omega / d k$ for the group velocity obtained from linear-dispersion theory. Also, the experimental results are quantitatively explained by adiabatic following, in which the pseudomoments of the atoms remain aligned along the effective field of the laser light. The adiabatic-following model allows for a direct comparison of our results with the work on self-induced transparency. For high-intensity light, adiabatic following predicts a nonlinear pulse velocity and the possibility of observing self-steepening.
\end{abstract}

\section{INTRODUCTION}

Many of the previous measurements of optical pulse velocity in resonant systems have been made by placing the material under study in a modelocked laser cavity and measuring the change in the pulse repetition rate. ${ }^{1-4}$ Using this technique, Faxvog et $a l .{ }^{3}$ measured pulse velocities exceeding $c$ by three parts in $10^{4}$, and Casperson and Yariv ${ }^{4}$ observed pulse velocities of $c / 2.5$. Apart from laser cavities, the coherent phenomenon of self-induced transparency ${ }^{5}$ can produce extremely slow pulse velocities for resonant pulses with an area of the order of $2 \pi$, and pulse velocities of $c / 1000$ have been measured. ${ }^{6}$ In this paper we report the observation of pulse velocities $v_{p}$ as slow as $v_{p}=\frac{1}{14} c$ in dilute rubidium vapor for short pulse of narrow-line dye-laser light nearly resonant with the $\sigma^{-}$component of the Zeeman-split ${ }^{2} P_{1 / 2}$ resonance line (7948 $\AA$ ) of rubidium.

In contrast to the earlier intracavity experiments, ${ }^{1-4}$ for our experiment the $\mathrm{Rb}$-vapor cell was completely passive and was located outside the laser. The frequency $\nu$ of the laser light was far out on the homogeneously broadened Lorentzian wing of the resonance line, which eliminated both saturation (hole burning) and the theoretical complications caused by working within the inhomogeneous line. We were able to observe directly the input pulses to the $\mathrm{Rb}$ cell and the delayed output pulses. The pulses were only slightly distorted after propagating through the vapor and were attenuated by the factor $e^{-\alpha z}$, where $\alpha$ was the smallsignal absorption coefficient on the Lorentzian wing and $z$ was the propagation distance into the $\mathrm{Rb}$ vapor.

In contrast to self-induced transparency, our results remained the same when the pulse width was changed from 3 to $18 \mathrm{nsec}$, when the intensity was changed by 400 , and when the input pulse areas were changed from $0.5 \pi$ to $50 \pi$. However, as in self-induced transparency, most of the energy in the propagating pulse was contained by the atoms as coherent excitation, the spatial extent of the pulse in the resonant material was reduced by the factor $v_{p} / c$, and the absorption was due to the relaxation processes which determined the homogeneous linewidth of the resonance line. Also, our observations confirmed that "the qualitative observation of a pulse delay beyond the input pulse tail is not sufficient evidence of (self-induced) transparency," as first discussed by Courtens and Szöke. ${ }^{7}$

\section{THEORY}

The experiments satisfied the two conditions required for adiabatic following.$^{8,9}$ First, $\delta \nu \ll\left|\nu-\nu_{0}\right|$, where $\delta \nu$ was the linewidth of the input light with frequency $\nu$ and $\nu_{0}$ was the resonant atomic frequency. Second, the pulse width was short compared to $T_{1}$ and $T_{2}$ of the atomic system. ${ }^{10}$ Thus, in the rotating frame the pseudomoments $\vec{p}$ of each $\mathrm{Rb}$ atom remained aligned along the changing effective field $\overrightarrow{\mathcal{E}}_{e}$ of the input laser pulse, as shown in Fig. 1. Consequently, the in-phase component $u$ of the macroscopic polarization $\overrightarrow{\mathrm{P}}$ of the $\mathrm{Rb}$ vapor was given by ${ }^{9,11,12}$

$$
u= \pm N_{e}|\overrightarrow{\mathrm{p}}| \sin \theta^{\prime}
$$

where the + sign is used for $\nu<\nu_{0}$, the - sign for $\nu_{0}<\nu$, and

$$
\sin \theta^{\prime}=\sqrt{2} E /\left(E_{s}^{2}+2 E^{2}\right)^{1 / 2}
$$

$\theta^{\prime}$ is the angle between $\overrightarrow{\mathrm{p}}$ and the negative $3^{\prime}$ axis; $N_{e}$ is the effective number density of atoms; $E$ is the electric field strength of the input circularly polarized light; $|\overrightarrow{\mathrm{p}}|=p_{12} / \sqrt{2}$, where $p_{12}$ is the absolute value of the matrix element for the electricdipole moment for the $\sigma$ transition $\left(p_{12}=6.16\right.$ $\times 10^{-18}$ esu for the ${ }^{2} P_{1 / 2}$ line of $\left.\mathrm{Rb}\right) ; E_{s}=\mid h\left(\nu-\nu_{0}\right) /$ 


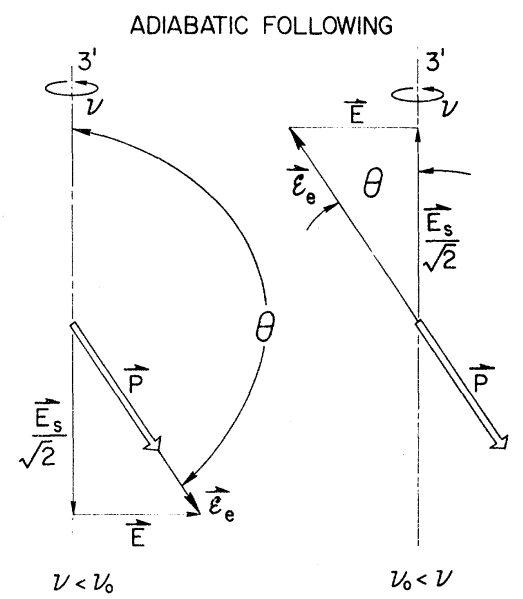

FIG. 1. Adiabatic following of the effective field $\overrightarrow{\mathcal{E}}_{e}$ (in the rotating frame in which $\overrightarrow{\mathrm{E}}$ appears stationary) by the pseudomoment $\vec{p}$, when the frequency $\nu$ of the input laser light is either less than or greater than the atomic resonant frequency $\nu_{0} . \quad \overrightarrow{\mathrm{E}}$ is the electric field of the input circularly polarized light; $|\vec{p}|=p_{12} / \sqrt{2}$, where $p_{12}$ is the absolute value of the matrix element of the electric-dipole moment for the $\sigma$ transition; $E_{s}=\left|h\left(\nu-\nu_{0}\right)\right|$ $p_{12} l$.

$p_{12} \mid . \quad N_{e} \overrightarrow{\mathrm{p}}$ is the pseudo-electric-dipole-moment of Abella; Kurnit, and Hartmann, ${ }^{13}$ and $\overrightarrow{\mathrm{p}}=p_{12} \overrightarrow{\mathrm{r}} /$ $\sqrt{2}$, where $\overrightarrow{\mathrm{r}}$ is the 3 -vector of Feynman, Vernon, and Hellwarth. ${ }^{14}$ Equation (1) can be rewritten as

$$
u=\frac{-N_{e} p_{12}^{2} E}{h\left(\nu-\nu_{0}\right)\left(1+2 E^{2} / E_{s}^{2}\right)^{1 / 2}} .
$$

Using Eq. (3) the real part of the nonlinear dielectric constant of the $R b$ vapor is obtained $a^{9}$

$$
\epsilon=1-\frac{4 \pi N_{e} p_{12}^{2}}{h\left(\nu-\nu_{0}\right)\left(1+2 E^{2} / E_{s}^{2}\right)^{1 / 2}} .
$$

For $E^{2} \ll E_{s}^{2}$, $\epsilon$ becomes

$$
\epsilon_{0}=1-\frac{4 \pi N_{e} p_{12}^{2}}{h\left(\nu-\nu_{0}\right)}
$$

which is the usual small-signal result.

For adiabatic following $\delta \nu \ll\left|\nu-\nu_{0}\right|$, which in turn implies that the out-of-phase component of the polarization $v$ is much smaller than the in-phase component $u$. Because $v \ll u$, the angle between the pseudomoment $\overrightarrow{\mathrm{p}}$ and the effective field $\overrightarrow{\mathscr{E}}_{e}$ is extremely small. Thus, to a very good approximation, we can consider $\overrightarrow{\mathrm{p}}$ to be parallel to $\vec{E}_{e}$, as shown in Fig. 1, when calculating $u$. Then, in the next order of approximation, we can use $u$ to calculate $v$.

The pulse velocity $v_{p}$ is easily derived from the Bloch equation relating $u$ and $v,{ }^{5}$

$$
\frac{\partial u}{\partial t}=-2 \pi v\left(\nu-\nu_{0}\right)-\frac{u}{T_{2}}
$$

and from the reduced wave equation ${ }^{5,6,15}$

$$
\frac{\partial E}{\partial z}+\left(\frac{\partial E}{\partial t}+4 \pi \frac{\partial u}{\partial t}\right) /\left(n_{0} c\right)=\frac{-(2 \pi)^{2} \nu v}{n_{0} c} \quad .
$$

Here $n_{0}=\sqrt{\epsilon_{0}}$, and $1 / T_{2}=1 / 2 \tau+1 / T_{c}$, where $\tau$ is the radiative lifetime of the excited state $(28 \mathrm{nsec}$ for the ${ }^{2} P_{1 / 2}$ line) and $T_{c}$ is the mean time between resonant collisions. ${ }^{16}$

Initially consider the situation where $T_{2} \rightarrow \infty$. For $u$ given by Eq. (3) $v$ is determined to be

$$
v=N_{e} p_{12}^{2} \frac{\cdot \partial E / \partial t}{2 \pi h\left(\nu-\nu_{0}\right)^{2}\left(1+2 E^{2} / E_{s}^{2}\right)^{3 / 2}} .
$$

Equation (8) shows that $v$ is much smaller than $u$, and our approximation scheme is self-consistent. Using this result for $v$, the reduced wave equation has simple wave solutions of the form ${ }^{17}$

$$
E(t, z)=E\left(t-z / v_{p}\right),
$$

where

$$
v_{p}=c /\left[\left(\epsilon+\frac{E d \epsilon}{d E}\right) / n_{0}+\nu\left(\frac{d n_{0}}{d \nu}\right)\left(1+\frac{2 E^{2}}{E_{s}^{2}}\right)^{-3 / 2}\right],
$$

with

$$
\frac{d n_{0}}{d \nu}=\frac{2 \pi N_{e} p_{12}^{2}}{n_{0} h\left(\nu-\nu_{0}\right)^{2}}
$$

The pulse velocity is nonlinear in the pulse intensity; the more intense parts of the pulse travel faster, and the leading edge should show selfsteepening. ${ }^{17,18}$ The simple wave solution of Eq. (9) describes this pulse reshaping until the solution becomes double valued, which is the analytical condition for the formation of an optical shock wave. We have observed self-steepening caused by this intensity-dependent pulse velocity.

However, our observations were complicated by self-defocusing ${ }^{9}$ and strong third-order mixing. Presently, we are in the process of reducing the strength of these processes so as to observe the clean formation of optical shocks. This work will be reported later.

For the experiments discussed in this paper $E^{2} \ll E_{s}^{2}$. Also, $\nu<\nu_{0}$ to eliminate the complicating effects of self-focusing. ${ }^{8}$ However, some data were taken with $\nu_{0}<\nu$ and slow pulse velocities were observed which were in relatively good agreement with theory. Now, consider finite $T_{2}$, and let $E^{2} \ll E_{s}^{2}$. For this case $u$ is obtained from Eq. (3) as

$$
u=\frac{-N_{e} p_{12}^{2} E}{h\left(\nu-\nu_{0}\right)},
$$

and $v$ is given by

$$
v=\left(\frac{N_{e} p_{12}^{2}}{2 \pi h\left(\nu-\nu_{0}\right)^{2}}\right)\left(\frac{\partial E}{\partial t}+\frac{E}{T_{2}}\right) .
$$

Using this result for $v$, the reduced wave equation 
again has simple wave solutions, but this time of the form

$$
E(t, z)=E\left(t-z / v_{p}\right) e^{-\alpha z / 2} .
$$

The pulse velocity is determined to be

$$
v_{p}=\frac{c}{n_{0}+\nu d n_{0} / d \nu} ;
$$

$d n_{0} / d \nu$ is given by Eq. $(11)^{19}$; the absorption coefficient $\alpha$ is equal to

$$
\alpha=\frac{2 \nu\left(d n_{0} / d \nu\right)}{c T_{2}}
$$

Equation (15) can be rewritten in the common form

$$
v_{p}=\frac{d \omega}{d k}
$$

where $\omega=2 \pi \nu$ and $k=2 \pi \nu n_{0} / c$.

Equations (15) and (16) are the same as the classical results for the group velocity and the absorption coefficient, obtained from linear-dispersion theory. ${ }^{20-24}$ This is not surprising, because for the experiment considered here $E^{2} \ll E_{s}^{2}$ (the angle $\theta^{\prime}$ between $\vec{p}$ and the $3^{\prime}$ axis remains very small) and saturation can be neglected, so that the equation of motion reduces to the equation of a damped harmonic oscillator. For adiabatic following the oscillator is excited at the driving frequency $\nu$ without excitation of transient oscillation at the resonant frequency $\nu_{0}$. Alternatively, this problem could be presented within the formalism of polaritons. ${ }^{25,26}$ The atomic vapor would be considered as a collection of randomly distributed independent oscillators. In this picture the normal modes of the resulting system would have a wavelike character for long wavelengths, the motion of the oscillators being correlated by the interaction between the oscillators and the traveling electromagnetic wave.

Courtens ${ }^{27}$ has given the expression for the pulse velocity in coherent propagation:

$$
v_{p}=\frac{c}{n_{0}+n_{0} U_{c} / U_{\mathrm{em}}} .
$$

Here $U_{c}$ is the energy density of coherent atomic excitation and $U_{\text {em }}$ is the energy density in the electromagnetic wave. This expression also applies to adiabatic following. With $\nu<\nu_{0}$ we obtain $U_{c}$ from Fig. 1 as

$$
U_{c}=\frac{1}{2}\left[h \nu_{0} N_{e}\left(1-\cos \theta^{\prime}\right)\right],
$$

where

$$
\cos \theta^{\prime}=\frac{E_{s}}{\left(E_{s}^{2}+2 E^{2}\right)^{1 / 2}} .
$$

Alternatively, $U_{c}$ can be obtained from the Bloch equation, which relates the $3^{\prime}$ component of the pseudomoment with the out-of-phase component of the polarization $v$. For $T_{1} \rightarrow \infty$ this equation can be written as $^{5}$

$$
\frac{\partial U_{c}}{\partial t}=2 \pi \nu_{0} v E
$$

Using $v$ given by Eq. (8), we can integrate Eq. (21) and obtain $U_{c}$ given by Eq. (19). This shows that the approximation method used to calculate $v$ conserves energy. For our experiment the angle $\theta^{\prime}$ between the pseudomoment and the negative $3^{\prime}$ axis is very small; consequently,

$$
U_{c}=h \nu_{0} N_{e}\left(\frac{1}{2} \theta^{\prime}\right)^{2},
$$

with $\theta^{\prime}=\sqrt{2} E / E_{s}$. For our situation the electromagnetic energy density is $U_{\mathrm{em}}=\epsilon_{0} E^{2} / 4 \pi$. For $\nu \sim \nu_{0}$, Courtens's equation agrees with Eq. (15).

It is quite instructive to derive the absorption coefficient $\alpha$ by intuitive arguments. For any point on the pulse envelope as the pulse propagates through the $R b$ vapor the total energy density $U=U_{c}+U_{\text {em }}$. We assume the only loss mechanism is spontaneous emission (with lifetime $\tau$ ) from $U_{c}$; consequently,

$$
\frac{\partial U}{\partial t}=\frac{-U_{c}}{\tau}
$$

Using Eq. (18), we can rewrite Eq. (23) as

$$
\frac{\partial U}{\partial t}=\frac{-U\left(c-n_{0} v_{p}\right)}{c \tau}
$$

or, equivalently,

$$
\frac{\partial U}{\partial z}=\frac{-U\left(c-n_{0} v_{p}\right)}{c \tau v_{p}},
$$

since the pulse velocity is $v_{p}$. This equation integrates to

$$
U=U_{0} e^{-\alpha z}
$$

where $z$ is the distance propagated into the vapor, $U_{0}$ is the energy density in the vapor at $z=0$, and

$$
\alpha=\left(1 / v_{p}-n_{0} / c\right)(1 / \tau)
$$

which is the same as Eq. (16) with $T_{2}=2 \tau$. Equation (27) shows that $\alpha$ is proportional to the difference between the reciprocals of the pulse velocity and the phase velocity. ${ }^{7}$

\section{EXPERIMENT}

The experimental arrangement shown in Fig. 2 is similar to that used in Ref. 9 , in which the rubylaser pumped dye laser and the $\mathrm{Rb}$ cell were described. Two Fabry-Perot interferograms were taken of each pulse. Both Fabry-Perot (FP) interferometers had a finesse of about 100. FP No. 1 had a spectral range of $0.33 \mathrm{~cm}^{-1}$ and FP No. 2 a spectral range of $2.5 \mathrm{~cm}^{-1}$. FP No. 1 monitored the linewidth $\delta \nu$ [Fig. 3(a)], while FP No. 2 measured the frequency difference between dye-laser 


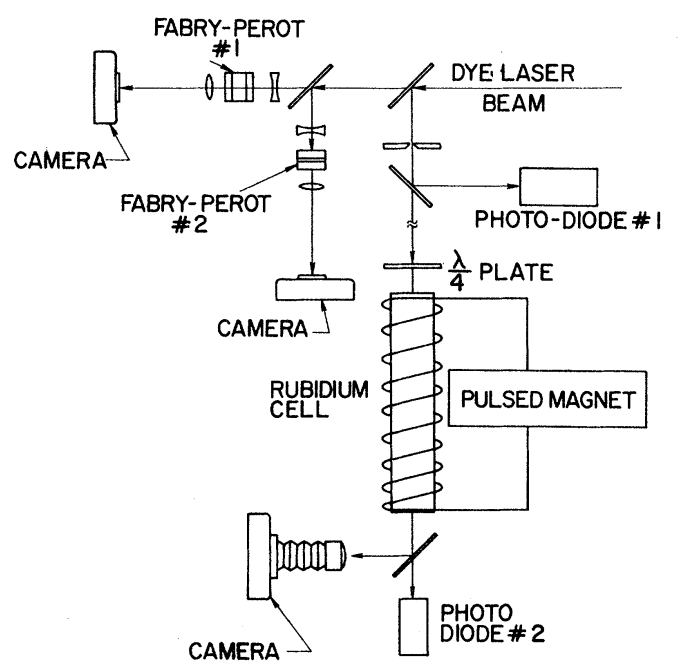

FIG. 2. Schematic diagram of the experiment.

output and the ${ }^{2} P_{1 / 2}$ line of a Rb spectral lamp. The main beam went through a 1-mm-diam aperture, $525 \mathrm{~cm}$ from the entrance window of the Rb cell. Consequently, the intensity profile of the beam that went through the $\mathrm{Rb}$ cell was the Fraunhofer diffraction pattern of the aperture. The input pulse [Fig. 3(b)] to the $\mathrm{Rb}$ cell was monitored with the ITT biplanar photodiode No. 1 connected to a Tektronix 519 oscilloscope. The main beam went through a quarter-wave plate, which determined the ratio of $\sigma^{+}$to $\sigma^{-}$light. After passing through attenuating filters, the pulse propagated through the $100-\mathrm{cm} \mathrm{Rb}$ cell while a variable pulsed magnetic field of, typically, $8 \mathrm{kG}$ was applied to the cell. A near-field photograph was taken of the beam at the exit window of the cell to check for self-defocusing. The output pulse [Fig. 3(c)] was observed with the ITT biplanar photodiode No. 2 connected to a Tektronix 7904 oscilloscope. For some experiments FP interferograms were taken of the emergent beam. At the low intensities used in the experiment (typically $40 \mathrm{~W} / \mathrm{cm}^{2}$ ) no selfdefocusing took place, nor was the frequency of the output beam broadened.

\section{EXPERIMENTAL RESULTS}

As shown in Fig. 3(c), a single pulse of linearly polarized dye-laser light was split into two pulses of $\sigma^{+}$and $\sigma^{-}$circularly polarized light by passage through the $100-\mathrm{cm}$ cell containing $\mathrm{Rb}$ vapor at a temperature of $140^{\circ} \mathrm{C}$ and a pressure of $2.3 \times 10^{-3}$ Torr and in a magnetic field of $8.5 \mathrm{kG}$. The frequency of the laser light was $0.25 \mathrm{~cm}^{-1}$ below the center frequency of the $\sigma^{-}$hyperfine components of the Zeeman-split ${ }^{2} P_{1 / 2}$ resonance line and 1.30 $\mathrm{cm}^{-1}$ below the center of the $\sigma^{+}$components. The $\sigma^{+}$pulse was not distorted by passage through the
$\mathrm{Rb}$ vapor, while the $\sigma^{-}$pulse was slightly changed. If the input pulse was circularly polarized, only the $\sigma^{+}$or the $\sigma^{-}$pulse was seen. Switching from one to the other was accomplished by rotating a quarter-wave plate (shown in Fig. 2) by $90 \mathrm{deg}$. Knowing that the transit time of the $\sigma^{+}$pulse through the $100-\mathrm{cm} \mathrm{Rb}$ cell was $\sim 4.2 \mathrm{nsec}$, then from the 26-nsec separation between the $\sigma^{+}$and $\sigma^{-}$pulses we can determine that the $\sigma^{-}$pulse velocity was $v_{p}^{-}=\frac{1}{9} c$. Also, we can determine that the absorption coefficient for the $\sigma^{-}$pulse was $\alpha=1.2 \times 10^{-2}$ $\mathrm{cm}^{-1}$ from the relative heights of the $\sigma^{+}$and $\sigma^{-}$ pulses.

The measured pulse velocities of the $\sigma^{-}$pulses through the $\mathrm{Rb}$ vapor are compared with Eq. (15) in Fig. 4; the $\sigma^{-}$pulse of Fig. 3 is shown by the open square. For this comparison it was impor-

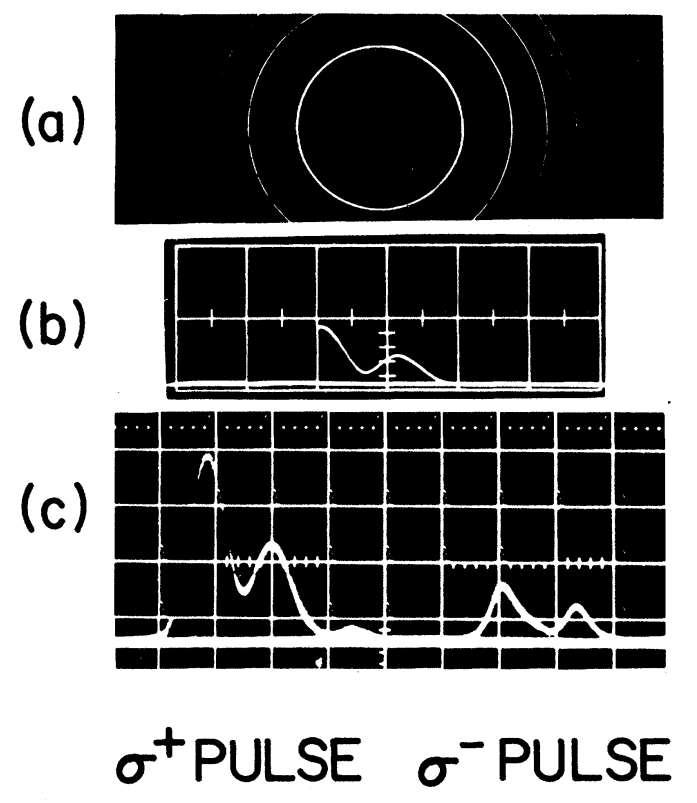

FIG. 3. (a) Fabry-Perot interferogram of the dyelaser pulse shown in (b) and (c). The interferometer had a finesse of $\sim 100$ and a spectral range of $0.33 \mathrm{~cm}^{-1}$. The measured linewidth $\delta \nu / c$ was less than $0.005 \mathrm{~cm}^{-1}$. (b) Linearly polarized input pulse to the Rb cell. The pulse was observed with an ITT biplanar photodiode and a Tektronix 519 oscilloscope. The sweep speed was 5 nsec per division and the peak power corresponded to $7 \mathrm{~W}$ entering the Rb cell. (c) Resulting two circularly polarized $\sigma^{+}$and $\sigma^{-}$output pulses from the $100-\mathrm{cm} \mathrm{Rb}$ cell. The pulses were observed with an ITT biplanar photodiode and a Tektronix 7904 oscilloscope. The sweep was $5 \mathrm{nsec}$ per division, and the measured pulse separation was $26 \mathrm{nsec}$, corresponding to a pulse velocity of $\frac{1}{9} c$ for the $\sigma^{-}$pulse. The Rb cell temperature was $140^{\circ} \mathrm{C}$, and the cell was in a magnetic field of $8.5 \mathrm{kG}$. The frequency of the laser light was $0.25 \mathrm{~cm}^{-1}$ below the center frequency of the $\sigma^{-}$hyperfine components of the Zeemansplit ${ }^{2} P_{1 / 2}$ resonance line of $\mathrm{Rb}$ and $1.30 \mathrm{~cm}^{-1}$ below the center of the $\sigma^{+}$components. 
tant to take into account the hyperfine splitting (in the Paschen-Bach limit) of the $\sigma$ components of the ${ }^{2} P_{1 / 2}$ line of the two isotopes of natural $\mathrm{Rb} .{ }^{6}$ For the data of Fig. 4, $\nu_{0}-\nu$ was known to about $\pm 0.02 \mathrm{~cm}^{-1}$; the pulse delays were measured to approximately $\pm 1 \mathrm{nsec}$ for large delays and \pm 0.5 nsec for small delays. A large part of the scatter of the data was due to the $\pm 1{ }^{\circ} \mathrm{C}$ temperature fluctuations of the $\mathrm{Rb}$ cell. The total atomic number density $N=2 N_{e}$ was determined from an adjusted equation of state which gave the best agreement with the data. This procedure gave $N=0.55 \times 10^{13} / \mathrm{cm}^{3}$ at $100^{\circ} \mathrm{C}, N=1.8 \times 10^{13} / \mathrm{cm}^{3}$ at $120^{\circ} \mathrm{C}$, and $N=5.5$ $\times 10^{13} / \mathrm{cm}^{3}$ at $140^{\circ} \mathrm{C}$. Also, this procedure gave $N=2.3 \times 10^{13} / \mathrm{cm}^{3}$ at $124^{\circ} \mathrm{C}$, which agrees well with the value $N=2 \times 10^{13} / \mathrm{cm}^{3}$ used in Ref. 9. The agreement between the measured pulse velocities and the calculated values is quite satisfactory; the good agreement shows that the coherent atomic excitation $U_{c}$ was given by Eq. (22). Consequently, the pseudomoments were aligned along the effective field as predicted by adiabatic following.

\section{DISCUSSION}

The propagation of the $\sigma^{-}$pulse of Fig. 3(c) through the $100-\mathrm{cm} \mathrm{Rb}$ cell (with $T_{2} \rightarrow \infty$ ) is illustrated by Fig. 5. The input pulse shown in Fig. 5(a) can be approximately considered as two adjacent 5-nsec pulses. Roughly speaking, a 5-nsec pulse in air is $150 \mathrm{~cm}$ long. For adiabatic following the electromagnetic wave and the coherent excitation of the atoms are coupled together by the dielectric response of the atomic vapor, and a

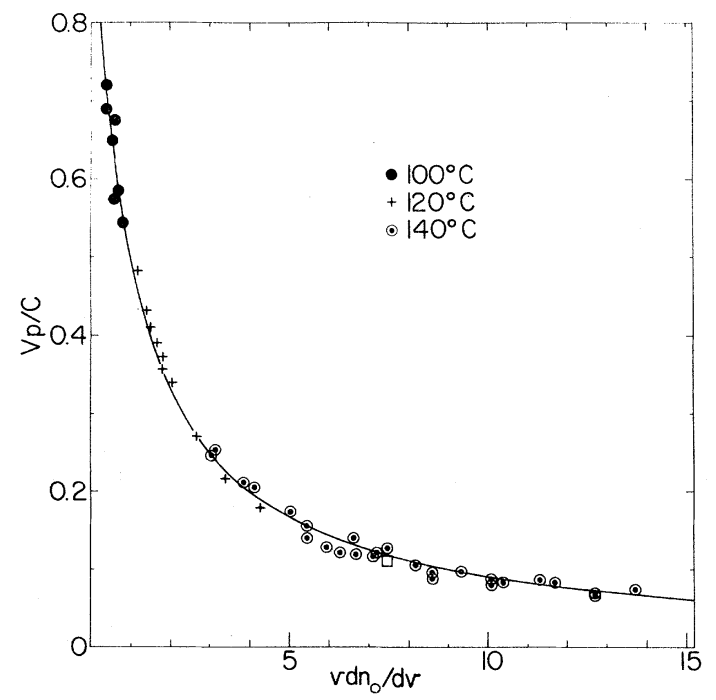

FIG. 4. Comparison with Eq. (15) of the measured pulse velocities of the $\sigma^{-}$pulses through the $\mathrm{Rb}$ vapor. Data were taken with the $\mathrm{Rb}$-vapor cell temperature at 100,120 , and $140^{\circ} \mathrm{C}$. The $\sigma^{-}$pulse of Fig. 3 is indicated by the open square.

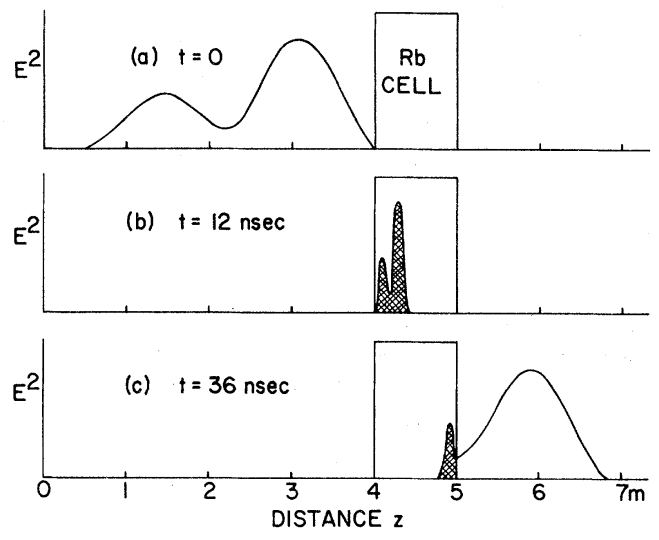

FIG. 5. Pictorial representation of the passage of the $\sigma^{-}$pulse of Fig. 3 through the $1-\mathrm{m}$ Rb cell at three different times. (a) Pulse entering the cell-designated as $t=0$. (b) $t=12 \mathrm{nsec}$. The pulse is completely within the vapor cell, traveling at $\frac{1}{9} c$, and has been compressed spatially by 9 . The cross hatching under the pulse envelope represents the energy density $U_{c}$ of the coherent excitation. (c) $t=36 \mathrm{nsec}$. The pulse is emerging from the vapor cell.

wave of excitation moves through the vapor in step with the electromagnetic wave, as indicated in Fig. 5(b). In the vapor the pulse velocity is $\frac{1}{9} c$ and $U_{c}=8 U_{\mathrm{em}}$. Thus, while the pulse is in the vapor cell only one-ninth of the total energy is in the electromagnetic wave; the other eight-ninths of the energy is in the coherent atomic excitation represented by the cross hatching under the pulse envelope. When the $\sigma^{-}$double pulse enters the $\mathbf{R b}$ vapor cell it is compressed by $v_{p}^{-} / c=\frac{1}{9}$ into two 16. 7- $\mathrm{cm}$ pulses. Thus, the cell is about six pulse lengths long, and most of the energy is transferred back and forth between the Rb atoms and the electromagnetic wave six times as the double pulse propagates through the cell. Because $\left(\epsilon_{0}-1\right) \ll 1$, the intensity of the pulse is unchanged upon entering the vapor. Also, even though the pulse is compressed, at any point in the cell the electromagnetic pulse, as a function of time, is the same as the input pulse. The transfer of energy between $U_{c}$ and $U_{\mathrm{em}}$, which occurs while the double pulse propagates through the cell, is accomplished without loss. The observed loss [shown in Fig. 3(c) but not in Fig. 5] is accounted for by the $T_{2}$ relaxation process.

If the relaxation processes could be completely neglected, the adiabatic-following model predicts that no energy would be lost to the propagating wave. The pulse would travel slowly without absorption or reshaping through the vapor. After the pulse went through the vapor there would be no residual excitation left in the atoms. A situation of complete transparency would occur for any pulse 
which satisfied the adiabatic-following conditions. This statement is easily understood in terms of the vector model. For adiabatic following as shown in Fig. 1 the pseudomoments remain aligned along the effective field. Initially, the pseudomoments and the effective field are pointing along the $3^{\prime}$ axis in the negative direction; all the atoms are in the electronic ground state. Then, as the pulse is applied, the pseudomoments and the effective field swing away from the $3^{\prime}$ axis, and coherent excitation energy is transferred to the atoms. Finally, when the pulse passes by, the pseudomoments and the effective field swing back to along the $3^{\prime}$ axis once again, and all the atomic excitation energy is returned to the propagating wave. This situation is completely determined by the motion of the effective field and is independent of inhomogeneous broadening, pulse width, and intensity; no special propagation properties are conferred by particular pulse areas or pulse shapes. For example, when the frequency is far enough off resonance to be in the adiabatic-following limit, the $2 \pi$ hyperbolic-secant pulse of self-induced transparency propagates no differently from any other pulse. ${ }^{28}$

Compared to adiabatic following, for self-induced transparency the pseudomoment motion is completely different (see Fig. 5 of Ref. 5). The effective field changes nonadiabatically, and consequently the pseudomoments make large angles with the effective field during the pulse. Self-induced transparency occurs when the motions of all the pseudomoments are such that when the pulse disappears they are all pointing in their original direction along the precession axis; otherwise excitation is left in the atomic system. This requirement is uniquely satisfied by the $2 \pi$ hyperbolicsecant pulse. ${ }^{5}$

Note added in proof. E. Courtens (private communication) has pointed out that in the derivation of Eq. (10) for the nonlinear pulse velocity it is unrealistic to neglect changes in the instantaneous frequency of the pulse caused by self-phase modulation. When self-phase modulation is included in the analysis, the pulse no longer propagates as a simple wave. Also, substantial frequency shifts are obtained, which play an important role in the formation of the optical shock.

M. D. Crisp (private communication) has discovered a novel approximation scheme which describes the nonlinear response of an atom to nearresonant light. The adiabatic-following model is obtained in the second order of approximation together with a closed-form expression for the error. Consequently, the range of validity of the adiabaticfollowing approximation can now be studied.

\section{ACKNOWLEDGMENTS}

The author would like to acknowledge that the simple wave solution with the nonlinear pulse velocity was outlined by J. A. Armstrong, and that N. S. Shiren correctly explained the preliminary experimental results. Useful discussions were held with E. Courtens, M. D. Crisp, Michael M. T. Loy, J. F. Reintjes, and P. P. Sorokin. The author would like to thank P. S. Zory for the generous loan of a Tektronix 7904 oscilloscope used in the inital experiments. Finally, R. J. Bennett made many important contributions to all phases of the experiment and the data presentation.
${ }^{1}$ J. A. Carruthers and T. Bieber, J. Appl. Phys. 40, 426 (1969).

${ }^{2}$ A. Frova, M. A. Duguay, C. G. B. Garrett, and S. L. McCall, J. Appl. Phys. 40, 3969 (1969).

${ }^{3}$ F. R. Faxvog, C. N. Y. Chow, T. Bieber, and J. A. Carruthers, Appl. Phys. Lett. 17, 192 (1970).

${ }^{4}$ L. Casperson and A. Yariv, Phys. Rev. Lett. 26, 293 (1971).

${ }^{5}$ S. L. McCall and E. L. Hahn, Phys. Rev. 183, 457 (1969).

${ }^{6}$ R. E. Slusher and H. M. Gibbs, Phys. Rev. A 5, 1634 (1972).

${ }^{7}$ E. Courtens and A. Szöke, Phys. Lett. 28A, 226 (1968)

${ }^{8}$ D. Grischkowsky, Phys. Rev. Lett. 24, 866 (1970).

${ }^{9}$ D. Grischkowsky and J. A. Armstrong, Phys. Rev. A 6, 1566 (1972)

${ }^{10} \mathrm{For}$ temperatures below about $140^{\circ} \mathrm{C}$ the homogeneous linewidth of the ${ }^{2} P_{1 / 2}$ transition is not broadened by resonant collisions (Ref. 16) but is determined by the lifetime $\tau=28$ nsec of the excited state. Because of severe radiative trapping, $\tau<T_{1}$. Below $140^{\circ} \mathrm{C}, T_{2}=2 \tau$. At $140^{\circ} \mathrm{C}, T_{2}$ was determined from Fig. 3(c) as $T_{2} \sim 45$ nsec, which indicated a slight amount of collision broadening. For the work presented in this paper the maximum dye-laser pulse width was $18 \mathrm{nsec}$, $\delta \nu / c<0.005 \mathrm{~cm}^{-1}$, and $\left|\nu-\nu_{0}\right| / c>0.15 \mathrm{~cm}^{-1}$; consequently, to a good approximation the conditions for adiabatic following were satisfied.

${ }^{11}$ I. I. Rabi, N. F. Ramsey, and J. Schwinger, Rev. Mod. Phys. 26, 167 (1954).

${ }^{12}$ M. D. Crisp, Phys. Rev. Lett. 22, 820 (1969); note in particular Eq. (13a).

${ }^{13}$ I. D. Abella, N. A. Kurnit, and S. R. Hartmann, Phys. Rev. 141, 391 (1966).

${ }^{14}$ R. P. Feynman, F. L. Vernon, and R. W. Hellwarth, J. Appl. Phys. 28, 49 (1957).

${ }^{15}$ Our equation (7) for the reduced wave equation differs from the corresponding Eq. (9) of Ref. 6 by the sign of the $\dot{u}$ term and by the presence of the low-intensity index of refraction $n_{0}$, which appears because we define the magnitude of the wave vector $k$ as $k=2 \pi n_{0} / \lambda$; in Ref. 6 the wave vector is $k=2 \pi / \lambda$.

${ }^{16}$ Ch'en Shang-Yi, Phys. Rev. 58, 884 (1940).

${ }^{17} \mathrm{~V}$. M. Arutyunyan, N. N. Badalyan, V. A. Iradyan, and M. E. Movsesyan, Zh. Eksp. Teor. Fiz. 58, 37 (1970) [Sov. Phys.-JETP 31, 22 (1970)]. Note in particular Eqs. (6) and (7). Equation (6) gives an expression for the nonlinear pulse velocity, and Eq. (7) gives the simple wave solution together with the distortions of phase caused by the nonlinear dielectric constant.

${ }^{18}$ F. DeMartini, C. H. Townes, T. K. Gustafson, and P. L. Kelley, Phys. Rev. 164, 312 (1967). 
${ }^{19}$ It should be noted that Eq. (15) predicts (in agreement with our observations) the same pulse velocity if only the sign of $v-v_{0}$ is changed.

${ }^{20} \mathrm{~L}$. Brillouin, Wave Propagation and Group Velocity (Academic, New York, 1960), p. 119.

${ }^{21}$ A. C. G. Mitchell and M. W. Zemansky, Resonance Radiation and Excited Atoms, 2nd ed. (Cambridge U. P., London, 1961), p. 128.

${ }^{22}$ E. O. Schulz-DuBois, Proc. IEEE 57, 1748 (1969).

${ }^{23}$ C. G. B. Garrett and D. E. McCumber, Phys. Rev. A 1, 305 (1970).

${ }^{24}$ M. D. Crisp, Phys. Rev. A 4, 2104 (1971).

${ }^{25}$ F. T. Arecchi, G. L. Masserini, and P. Schwendimann, Riv.
Nuovo Cimento 1, 181 (1969).

${ }^{26}$ D. L. Mills and A. A. Maradudin, Phys. Rev. B 1, 903 (1970).

${ }^{27}$ E. Courtens, Phys. Rev. Lett. 21, 3 (1968).

${ }^{28}$ For the $2 \pi$ hyperbolic-secant pulse of Ref. 5 , when $1 / \tau \ll|\Delta \omega|$ and $T_{1}, T_{2} \rightarrow \infty$ where $\Delta \omega=2 \pi\left(\nu_{0}-v\right)$ and $\tau$ is the pulse width, Eqs. (52) and (53) of Ref. 5 for $u$ and $v$ become the same as Eqs. (12) and (13) of this paper; Eq. (54) for $W$ of Ref. 5, apart from the normalization factor $-\mathrm{N}_{e}(1 / 2)\left(\mathrm{h} \nu_{0}\right)$, reduces to Eq. (22) for $U_{c}$ of this paper.. Also, Eq. (56) of Ref. 5 for the reciprocal of the pulse velocity becomes equivalent to our equation (15).

\title{
Cascade Time of Negative $K$ Mesons in Liquid Deuterium* ${ }^{\dagger}$
}

\author{
R. A. Burnstein, D. P. Novak, $\ddagger$ H. A. Rubin, and V. R. Veirs ${ }^{\S}$ \\ Illinois Institute of Technology, Chicago, Illinois 60616
}

(Received 2 March 1972)

\begin{abstract}
The cascade time of $\mathrm{K}^{-}$mesons has been measured in liquid deuterium using a deuterium-filled bubble chamber as the particle detector and absorber. The experimental result of $T_{\text {cascade } \mathrm{D}_{2}}=6_{-6}^{+7} \times 10^{-12}$ $\mathrm{sec}$ is consistent with results for liquid hydrogen and with the prediction that "Stark-effect mixing" allows rapid capture from $s$ states of high principal quantum number $n$.
\end{abstract}

\section{INTRODUCTION}

The cascade or absorption times of negative $\pi$, $K$, and $\Sigma$ particles in liquid hydrogen have been measured using the bubble chamber as the detector and absorption medium by means of a technique reported previously. ${ }^{1-3}$

For hydrogen, the experimental results for $\pi$, $K$, and $\Sigma$ particles indicate that the cascade times are in the range of $(0-4) \times 10^{-12}$ sec. These results are consistent with the prediction of Day, Snow, and Sucher ${ }^{4,5}$ and the calculations of Leon and Bethe $^{6}$ that "Stark-effect mixing" leads to rapid capture from $s$ states of high principal quantum number $n$. However, this technique ${ }^{1-3}$ cannot be used to measure the cascade time of antiprotons since the $\bar{p}$ is a stable particle.

There have been measurements of cascade times in liquid helium and liquid deuterium. In the case of liquid helium, experiments involving $\pi^{-}, K^{-}$, and $\Sigma^{-}$particles $^{7-9}$ have yielded cascade times $\sim 100$ times longer than comparable times in hydrogen. An explanation of these results is lacking but there are a number of speculations. ${ }^{10}$ It seems reasonable to conclude that, at least in part, the long cascade time for helium is due to the fact that the mesonic helium atom is not influenced appreciably by Stark-effect mixing leading to $s$-state capture. The electric fields due to neighboring atoms can have very different effects on the two types of mesonic or hyperonic atoms $\left(X^{-}, p\right)$ and
$\left(X^{-}, \mathrm{He}\right)$, where $X^{-}$is a negative meson or hyperon. In the $\left(X^{-}, p\right)$ case, the atom is neutral and can easily penetrate within a hydrogenic orbit, whereas in the $\left(X^{-}, \mathrm{He}\right)$ case, the mesonic or hyperonic atom has a net positive charge and cannot penetrate the Bohr orbits of neighboring atoms.

For the case of liquid deuterium, a similarity with hydrogen is expected since the mesonic or hyperonic atom would be neutral and the hydrogenic orbits penetrable. The one measurement of negative- $\pi$ capture times in deuterium ${ }^{11}$ is similar to measurements of $\pi$ capture times in hydrogen.

This paper is the first experimental study of the negative- $K$-meson cascade time in liquid deuterium: The experimental procedure is discussed in Sec. II and results and discussion follow in Sec. III.

\section{EXPERIMENTAL PROCEDURE}

The $K^{-}$mesons were produced by a two-stage electrostatically separated beam designed and built at the Brookhaven AGS accelerator to provide $\sim 600$ $1200 \mathrm{MeV} / c$ negative particles. The details and parameters of the beam are described elsewhere. ${ }^{12}$ In this experiment the $K^{-}$beam was transported at $750 \mathrm{MeV} / c$, then slowed down by a $110-\mathrm{cm}$ polyethylene moderator in front of the 30 -in. deuteriumfilled bubble chamber. The momentum of the beam as it entered the bubble chamber was $\sim 240 \pm 20$ $\mathrm{MeV} / c$. The run consisted of 37000 photographs with each frame containing 3-4 stopping $K$.

The specific experimental method used consists 


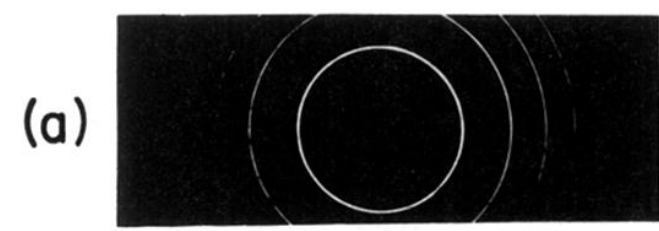

(b)
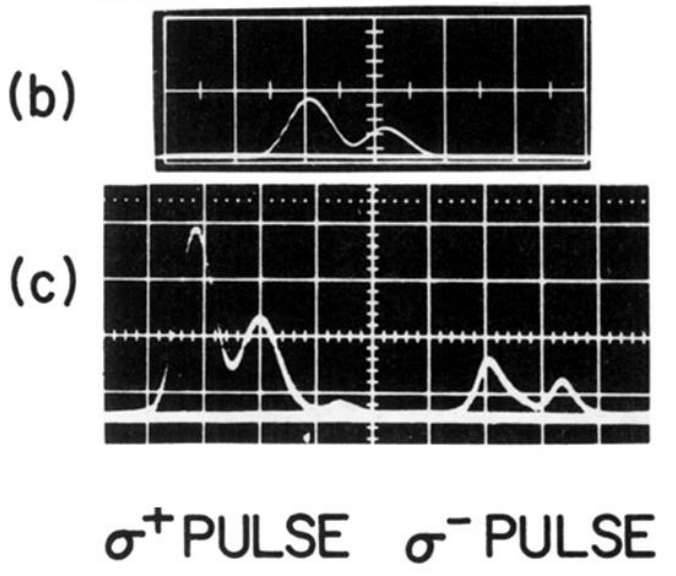

FIG. 3. (a) Fabry-Perot interferogram of the dyelaser pulse shown in (b) and (c). The interferometer had a finesse of $\sim 100$ and a spectral range of $0.33 \mathrm{~cm}^{-1}$. The measured linewidth $\delta \nu / c$ was less than $0.005 \mathrm{~cm}^{-1}$. (b) Linearly polarized input pulse to the $\mathrm{Rb}$ cell. The pulse was observed with an ITT biplanar photodiode and a Tektronix 519 oscilloscope. The sweep speed was 5 nsec per division and the peak power corresponded to $7 \mathrm{~W}$ entering the Rb cell. (c) Resulting two circularly polarized $\sigma^{+}$and $\sigma^{-}$output pulses from the $100-\mathrm{cm} \mathrm{Rb}$ cell. The pulses were observed with an ITT biplanar photodiode and a Tektronix 7904 oscilloscope. The sweep was 5 nsec per division, and the measured pulse separation was $26 \mathrm{nsec}$, corresponding to a pulse velocity of $\frac{1}{9} c$ for the $\sigma^{-}$pulse. The $\mathrm{Rb}$ cell temperature was $140^{\circ} \mathrm{C}$, and the cell was in a magnetic field of $8.5 \mathrm{kG}$. The frequency of the laser light was $0.25 \mathrm{~cm}^{-1}$ below the center frequency of the $\sigma^{-}$hyperfine components of the Zeemansplit ${ }^{2} P_{1 / 2}$ resonance line of $\mathrm{Rb}$ and $1.30 \mathrm{~cm}^{-1}$ below the center of the $\sigma^{+}$components. 